\title{
Starting Strategy of Thyristor Power Supply in Parallel Resonant Induction Heating on Heavy Load
}

\author{
Sen-Lin Cheng, $\mathrm{Hu} \mathrm{Xu}, \mathrm{Bin} \mathrm{Li}, \mathrm{Bu}-\mathrm{Qing}$ Liu and Chuan Wang \\ School of Automation, Chongqing University, Chongqing, China \\ csl@cqu.edu.cn,1597725908@qq.com,lzbymcqc@sohu.com, \\ 739364767@qq.com,499875183@qq.com
}

\begin{abstract}
On heavy load, the energy supplement in the starting process of Thyristor Power Supply for Parallel Resonant Induction Heating (TPSPRIH) has trouble in catching up its consumption which makes the system start more difficultly. Aimed at the puzzle, this paper proposes a starting strategy based on the analysis of parameters of heating inductor and starting circuit. Considered the process complexity in commonly used pre-charged circuit, it analyzed relationship among the parameters of heating inductor, the iron material and its filling status. Compared the supply speed with the decay characteristic of load circuit, the critical starting conditions of TPSPRIH on heavy load has been gotten, then derived the solving space of trigger angle of rectifier on energy providing. Based on the rated parameters of a typical system, the starting strategy of rectifier angle has been proposed. The simulation on different regions in the solving space has been made. The simulation result demonstrated the rationality and correctness. It probably provides a reference way for the reliable starting of TPSPRIH.
\end{abstract}

Keywords: Induction Heating, Thyristor Power Supply, Starting Strategy, Heavy Load

\section{Introduction}

Induction heating power supply is an energy conversion device, which is widely used in the forging industry $[1,2]$. The TPSPRIH raised more concern because it can provide more efficient power, but it's difficult to start on heavy load because of its big problem in its popularization and application, especially when it's starting on heavy load, the success rate of starting is extremely low [3-5]. Although many scholars have studied on it, such as the [6] studied the pre-charge starting, the [7] proposed the double auxiliary bridge starting, the [8] presented the load premagnetizing starting etc., but their starting methods are mainly by means of changing topology of circuit to increase the medium-frequency voltage when converting the current so as to improve the success rate of starting on heavy load.

In fact, if the energy supply speed of load circuit is not high enough, the success rate of starting is still very low although the supply voltage is at a high level. Study in engineering found that, in the process of starting, the decay and supplying speed of energy in load circuit, the state of load circuit [9-11] and the equivalent parameters of heating inductor [12-15] in different starting conditions affect each other, but a clearly comprehensive analysis of their interaction has not been found. In the paper, taken the commonly used pre-charge starting as an example, from the view of rectifier control angle, it established the objective function, studied on the characteristics of load circuit within the objective function on heavy load, and exported the control conditions in the starting process of TPSPRIH. 


\section{Zero-Input Response on Different Load}

The pre-charge starting is one of the forced starting methods, and there is a higher success rate of starting on heavy load under the same condition [16]. The scheme circuit of pre-charge starting is shown in Figure 1. $T_{1} \sim T_{5}$ are thyristors, $L_{d}$ is the inductance of reactor in DC side. $L, R, C, I_{d}, i_{H}$ and $u_{H}$ is respectively the equivalent inductance of heating inductor, the equivalent resistance of heating inductor, the compensation capacitor of load circuit, the current in DC side, the medium-frequency current and voltage of load circuit.

During starting process, the pre-charged $C_{P}$ through $T_{5}$ charges to the compensation capacitor $C$. Before the initial energy of load circuit decay to zero, the triggering thyristor $T_{1} \sim T_{4}$ conducts alternately, and at the same time, it triggers the rectifier bridge. When the medium - frequency voltage is established steadily on load circuit, it means to be started successfully.

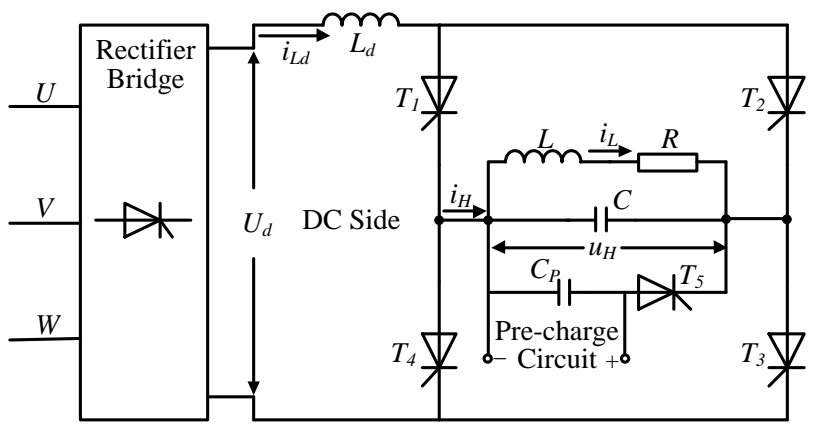

Figure 1. The Scheme Circuit of Pre-Charged Starting For TPSPRIH

In the above equivalent load circuit, the $L$ and $R$ of equivalent heating inductor are varied with the time varying. When it's starting on heavy load, the varying rate of $R$ and $L$ owns rather difference, it accelerates the energy decay speed on load circuit, it leads the energy decay speed to be far higher than its supplying, and therefore, it results in thyristor inverter becoming hard to convert current normally. The medium-frequency voltage on the load becomes hard to establish, and the start will be failed.

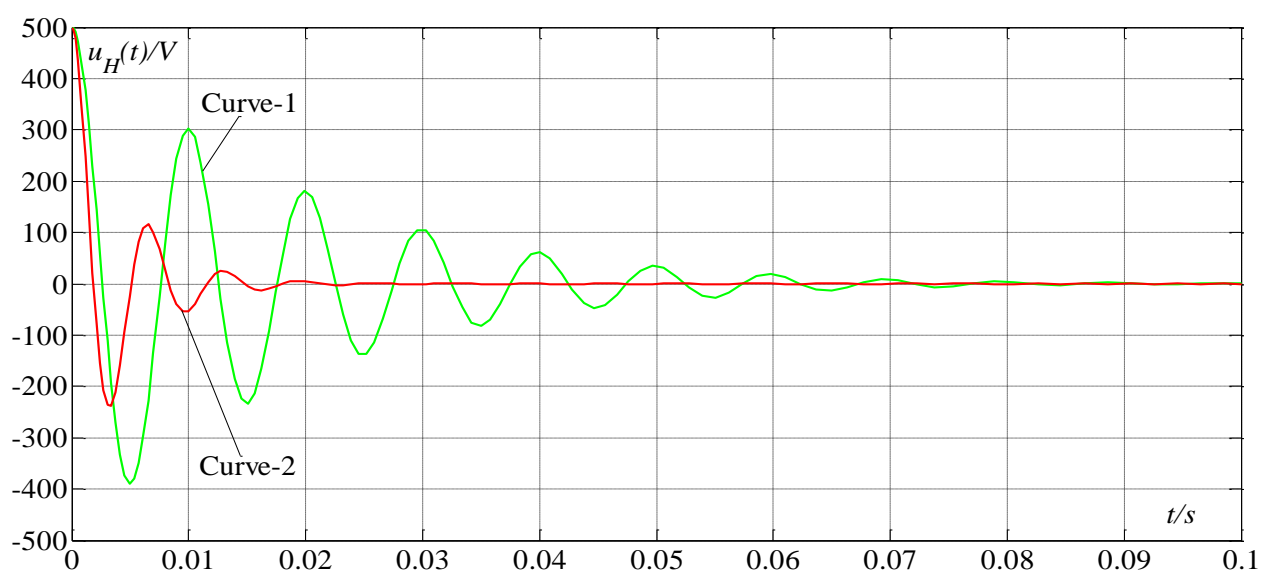

Figure 2. The Decay Curves on Different Load

The comparison response curves of load voltage in zero-input are shown in Figure 2. In the figure, the curve- 1 is the decay curve of load voltage on light load, and the curve- 2 is on heavy load. Both of them are at the same initial condition, and in which, their precharged voltages are $500 \mathrm{~V}$, but the decay speed of curve- 2 is faster than curve- 1 
obviously. The basic reason is that the variation rate of $R$ and $L$ is enormously different on heavy load, which causes the decaying coefficient of load circuit to be increased greatly, and therefore, it becomes more difficult to start.

\section{Selection of Control Parameter}

\subsection{Performance of Heating Inductor}

Heating inductor can be simplified as coil with iron core, and the equivalent circuit is shown as in Figure 3. Where, $R_{C u}, R_{F e}$ and $L$ is respectively the wire resistance, the equivalent resistance and equivalent inductance of heating inductor. Generally, the $R_{C u}$ is mall enough to be ignored, and therefore, $R_{F e}$ and $L$ are the key factors affecting the technical performance of heating inductor.

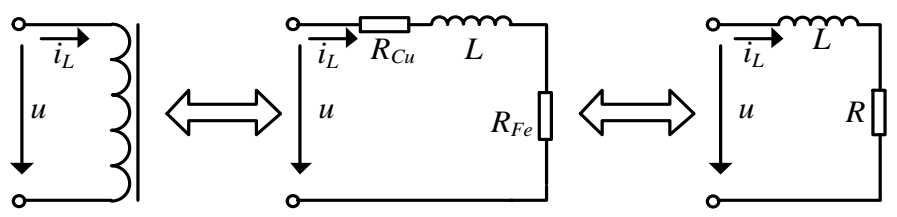

Figure 3. Equivalent Circuit of Heating Inductor

When the iron bar is heated, it is put into the heating inductor. The equivalent inductance and resistance is respectively shown as formula (1) and (2).

$$
\begin{gathered}
L=N \mu_{r} \mu_{0} n \pi r^{2} \\
R=R_{F e}
\end{gathered}
$$

Among them, $n=N / l, l, r, \mu_{0}$ and $\mu_{r}$ is respectively the turn number of coil in unit length, coil length, bar radius, permeability of vacuum and the differential permeability of iron bar. The $\mu_{r}-T$ curve is shown as in Figure 4, and in which, $T_{0}$ is the Curie temperature of iron bar.

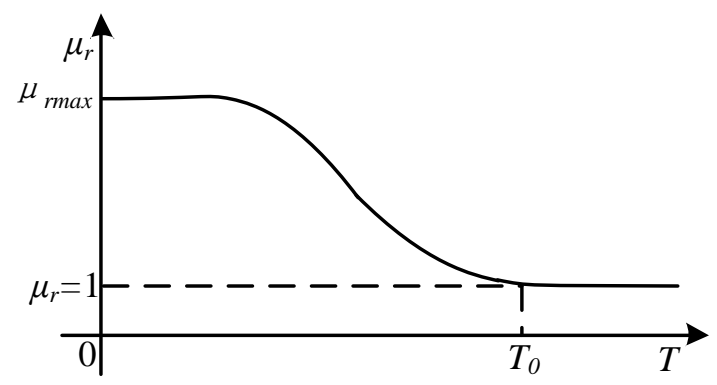

Figure 4. $\mu_{r}-T$ Curve

From Figure 4, when the heating inductor is unloaded, there must be $\mu_{r}=1$. When it's on heavy load, it exists $\mu_{r}=\mu_{r m a x}$. When it's on heavy load with high temperature (i.e. $T$ $\geqslant T_{0}$ ), it must meet $\mu_{r}=1$. From formula (1), when it's on heavy load to start with the physical size and other parameters of the heating inductor under the same condition, its equivalent inductance $L$ reaches to the maximum, and also the variation law of $L-T$ is the same as $\mu-T$ shown as in Figure 4.

Assuming that the turn number of heating inductor is $N$, and its current is $i_{L}(t)$ shown as in Figure 3, then the magnetic induction on the coil axis is expressed as formula (3).

$$
B=\mu N i_{L}(t)
$$


Within the heating inductor, before the temperature of heating material reaches its Curie temperature $T_{0}$, the iron loss includes the hysteresis loss and eddy current loss [17], and the power of hysteresis loss part $P_{h}$ can be expressed as following.

$$
P_{h}=K_{h} \omega B_{m}^{n} V
$$

The eddy current loss part $P_{e}$ is expressed as formula (5).

$$
P_{e}(t)=K_{e} \omega^{2} B_{m}^{2} V
$$

Where, $\omega, B_{m}, n, V, K_{h}, K_{e}$ is respectively the alternating frequency of magnetic field which is the same as the load oscillation frequency in the paper, the amplitude of magnetic induction intensity which is varying with formula (3), the exponential, the volume of heating material, the correlation coefficient of ferromagnetic materials which is determined by electrical resistivity, cross sectional area and shape which can be gotten from experiment.

Combined formula (4) and (5), the iron loss can be expressed as formula (6).

$$
P_{F e}(t)=K_{F e} \omega^{\beta} B_{m}^{2} V
$$

Where, $K_{F e}$ and $\beta$ are the constant, in which, $K_{F e}$ is determined by experiment, and generally, the $\beta$ takes the value from 1.2 to 1.6.

When the temperature of heated material in the heating inductor is higher than its Curie point $T_{0}$, the iron loss only includes the eddy current loss shown as formula (5). In the formula (6), when the iron loss and $i_{L}(t)$ are instantaneous value, it can get the resistance $R$ of heating inductor in DC circuit according to the thermal effect of resistance.

$$
R=R_{F e}=\frac{K_{F e} \omega^{\beta} B_{m}^{2} V}{i_{L}^{2}(t)}
$$

From formula (3), (6) and (7), it can be obtained that when the varying law of $i_{L}(t)$, heating material and initial trigger frequency of inverter are the same, the equivalent resistance $R$ of heating inductor owns the relationship $R \propto N \mu^{2}$, and the equivalent $L$ owns the relationship $L \propto \mu$ from formula (1). Now that the variation rate of $R$ is much faster than $L$ under the same limit condition, the decay factor of load circuit will increase rapidly because of the variation of heating inductor parameters $R$ and $L$, and therefore, it will lead to the fact that load energy decay is faster, and the starting on heavy load becomes more difficult.

But the formula (7) is only a kind of qualitative estimation of the variation of $R$, and in which, it is not applicable in engineering. Therefore, it is relatively accurate to the estimating value of $R$ based on the identification of oscillation frequency $\omega$ in the beginning of starting process.

$$
R=2 L \sqrt{\frac{1}{L C}-\omega^{2}}
$$

\subsection{Analysis of Load Circuit}

\subsubsection{Current of Load Circuit}

In the process of pre-charge starting, the initial trigger frequency of inverter is determined by the natural oscillation of load circuit. When it's on parallel resonance, the load current in Figure 1 must meet the expression (9).

$$
i_{L}(t)=Q i_{L d}(t) \sin \omega t
$$

Where, $Q$ is the quality factor of $R L C$ parallel resonant circuit, and it can be expressed as formula (10) approximately.

$$
Q=\frac{\omega L}{R}
$$

In the starting process, $i_{L}(t)$ consists of pre-charge decay oscillation current and supplying current of DC side, i.e. 


$$
i_{L}(t)=i_{L 0}(t)+\mathrm{Q} i_{L d}\left(t-t_{0}\right) \sin \omega\left(t-t_{0}\right)
$$

Where, $i_{L O}(t)$ is the pre-charge decay oscillation current, namely the current of the precharged energy to decay oscillation. $i_{L d}(t)$ is the supplying current of DC side, and $t_{0}$ is the lag trigger time of inverter.

It's necessary to analyze the decaying process of pre-charged energy when the load circuit is on zero-input state. And the load equivalent circuit is shown as the Figure 5. The load circuit energy consumption is totally represented by the power of resistance $R$, so the energy consumption speed of load circuit is the power on $R$.

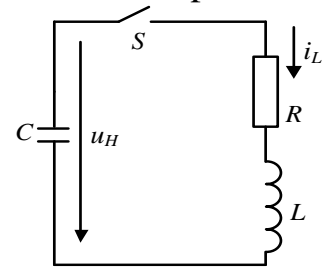

\section{Figure 5. Equivalent Load Circuit of Zero-Input Response}

In Figure 5, the initial pre-charged voltage of compensation capacitor $C$ is $U_{C 0}$, after closing switch $S$, the parameters of the circuit must meet $R<2 \sqrt{L / C}$, and the closed loop of $R L C$ would produce the decay oscillation.

$$
\begin{gathered}
L C \frac{d^{2} u_{H}(t)}{d t^{2}}+R C \frac{d u_{H}(t)}{d t}+u_{H}(t)=0 \\
i_{L 0}(t)=-C \frac{d u_{H}(t)}{d t}
\end{gathered}
$$

From (12) and (13), the formula (14) would be held.

$$
i_{L 0}(t)=\frac{U_{C 0}}{\omega L} e^{-\delta t} \sin \omega t
$$

In which,

$$
\begin{gathered}
\omega=\sqrt{\frac{1}{L C}-\delta^{2}} \\
\delta=\frac{R}{2 L}
\end{gathered}
$$

Where, $U_{C O}, \omega$ and $\delta$ is respectively the pre-charged voltage of $C$, frequency of medium - frequency decay oscillation, and the decay factor of medium - frequency voltage decaying which reflects the consumption speed of energy of load circuit.

In the starting process, assuming that the rectifier triggering time is the initial moment $(t=0)$, then the current $i_{L d}(t)$ through the reactor $L_{d}$ can be expressed as formula (17).

In which,

$$
i_{L d}(t)=I_{d 0}+I_{F}\left(1-e^{-t / \tau_{L_{d}}}\right)
$$

$$
\begin{gathered}
I_{\mathrm{F}}=\frac{U_{d}}{R_{1}} \\
\tau_{L d}=\frac{L_{d}}{R_{1}} \\
U_{d}=U_{2} f(\alpha) \\
R_{1}=\frac{L}{R C}
\end{gathered}
$$

Where, $I_{d 0}, I_{F}, U_{2}, U_{d}, \alpha, f(\alpha)$ and $R_{1}$ is respectively the initial current of $L_{d}$, expected steady state current, input phase effective voltage of rectifier, average voltage of rectifier output, trigger angle of rectifier, function reflecting the relationship of $U_{d}$, rectifier angle, and the equivalent oscillation resistance of load circuit. 
Integrating the formula (8), (11), (14) and (17), the current on heating inductor in starting process can be expressed as formula (22).

$$
i_{L}(t)=\frac{U_{C 0}}{\omega L} e^{-\delta t} \sin \omega t+Q\left[I_{d 0}+\frac{U_{2} f(\alpha)}{R_{1}}\left(1-e^{-\left(t-t_{0}\right) / \tau_{L_{d}}}\right)\right] \sin \omega\left(t-t_{0}\right)
$$

\subsubsection{Power of Load Circuit}

In the starting process, the instantaneous power of load circuit is expressed as formula (23).

$$
P_{\text {out }}(t)=i_{L}^{2}(t) R
$$

Combining with formula (22), it would get the expression (24).

$$
P_{\text {out }}(t)=\left(\frac{U_{C 0}}{\omega L} e^{-\delta t} \sin \omega t\right)^{2} R+\left\{Q\left[I_{d 0}+\frac{U_{2} f(\alpha)}{R_{1}}\left(1-e^{-\left(t-t_{0}\right) / \tau_{L_{d}}}\right)\right] \sin \omega\left(t-t_{0}\right)\right\}^{2} R
$$

Where, the front part of $P_{\text {out }}(t)$ is the consumption power of pre-charged energy, the latter one is the consumption of supplying energy, and compared with the latter, the front is negligible. Setting the trigger time of rectifier as $t=0$, expression (25) and (26) are the simplification of formula (11) and (24).

$$
\begin{gathered}
i_{L}(t)=Q i_{L d}(t) \sin \omega t \\
P_{\text {out }}(t)=\frac{\omega^{2} L^{2}}{R}\left[I_{d 0}+\frac{U_{2} f(\alpha)}{R_{1}}\left(1-e^{-t / \tau_{L d}}\right)\right]^{2} \sin ^{2} \omega t
\end{gathered}
$$

And the instantaneous supplying power of load circuit from DC side can be expressed as formula (27).

$$
P_{\text {in }}=U_{d} i_{L d}(t)
$$

Combining formula (17) with (20), the formula (28) can be derived.

$$
P_{i n}=U_{2} f(\alpha)\left[I_{d 0}+\frac{U_{2} f(\alpha)}{R_{1}}\left(1-e^{-t / \tau_{L_{d}}}\right)\right]
$$

\subsection{Starting Control of TPSPRIH}

There are many factors which could affect the starting process, but the initial current $I_{d 0}$ of the DC side, rectifier angle $\alpha$ and the initial trigger frequency $\omega_{1}$ of inverter are the most concerned factors in this paper.

\subsubsection{Selection of Initial Current $I_{d 0}$}

Because the initial current is too small, it would lead to start failure of the TPSPRIH on heavy load, and thus, it is necessary to select an appropriate initial current. There must be two crossing points in half-oscillation-period as $P_{\text {out }}(t)=P_{\text {in }}(t)$ in engineering, or there will be no difficulty in starting. Assuming that they crossed the second time at $t=t_{1}$. If the required voltage in the inverter convert process is $U_{0}$, then it can get the time $t_{1}$ concerning the limit condition.

$$
\begin{aligned}
t_{1} & =\frac{1}{4} T_{H} \\
T & =\frac{2 \pi}{\omega}
\end{aligned}
$$

Where, $T_{H}$ is the load oscillation period. And the medium frequency voltage of load circuit is expressed as formula (31).

$$
u_{H}\left(t_{1}\right)=\left.L \frac{d i_{L}(t)}{d t}\right|_{t=t_{1}}+R i_{L}\left(t_{1}\right)
$$

The thyristor converter must satisfy the basic conditions as formula (32) when converting current. 


$$
u_{H}\left(t_{1}\right) \geq U_{0}
$$

From formula (9), (21), (25) - (26) and (28) - (32), the minimum value of $I_{d 0}$ is expressed as formula (33).

$$
I_{d 0 \min }=\frac{U_{0} R L_{d}-U_{2} f(\alpha) \omega\left(R^{2} L_{d} C+L^{2} e^{-\frac{\pi L}{2 R L_{d} C \omega}}\right)}{R L_{d} L \omega}
$$

\subsubsection{Selection of Rectifier Angle $\alpha$}

When starting on heavy load, the energy supplying speed is crucial, at the same time, which is determined by $\alpha$. Usually it requires that, the smaller the angle is, the better the start performance. But the step voltage applied to the whole system is too large when $\alpha$ is small, it would produce instantaneous high voltage in the reactor which could be up to thousands or even tens of thousands volt. And it would break down the circuit system, on the other hand, if $\alpha$ is too large, it can not meet the requirements of starting. Therefore it is necessary to select an appropriate $\alpha$ to ensure the starting under the higher performance.

During the starting process, compared with the supplying energy, the pre-charged energy can be ignored. Thus the key to start successfully lies in the energy supplying speed, and in which, it can not less than its consumption speed.

$$
\begin{gathered}
P_{\text {in }} \geq P_{\text {out }} \\
u_{d}(\alpha)=\sqrt{6} U_{2} \sin \left(\frac{\pi}{3}+\alpha\right)
\end{gathered}
$$

Where, $u_{d}(\alpha)$ is the instantaneous voltage of three-phase rectifier output when its trigger angle is $\alpha$. Taking $P_{\text {in }}=P_{\text {out }}$, and when $\alpha$ is taken as the maximum value based on formula (26) and (28), it could get the Eq. (36).

$$
u_{d}\left(\alpha_{\max }\right) i_{L d}(t)=\frac{\omega^{2} L^{2}}{R}\left[i_{L d}(t)\right]^{2} \sin ^{2} \omega t
$$

The solution of formula (36) can be expressed as formula (37).

$$
\alpha_{\max }= \begin{cases}-\frac{\pi}{3}+\arcsin \left[\frac{\omega^{2} L^{2}}{\sqrt{6} R U_{2}} i_{L d}(t) \sin ^{2} \omega t\right], & 0 \leq \alpha \leq \frac{\pi}{6} \\ \frac{2 \pi}{3}-\arcsin \left[\frac{\omega^{2} L^{2}}{\sqrt{6} R U_{2}} i_{L d}(t) \sin ^{2} \omega t\right], & \frac{\pi}{6}<\alpha \leq \frac{7 \pi}{6}\end{cases}
$$

From formula (37), it can be derived that the maximum rectifier angle is determined by resonant frequency, current $i_{L d}(t)$ in DC side, and the parameters of load circuit. The above condition can ensure that whether it would be started successfully, but the starting performance is not necessary to be the optimal. Therefore, it is necessary to explore the optimal solution of $\alpha$ in the starting process, and which is decided by the designed safety voltage of the power system. If the safe voltage is $U_{s 0}$, the most voltage must be generated on the reactor in the changing process of current $i_{L d}(t)$.

Assuming $I_{d 0}=0$ in the formula (17), and combined with formula (19), it could derive out the change rate of current.

$$
\frac{d i_{L d}(t)}{d t}=\frac{u_{d}(\alpha)}{L_{d}} e^{\frac{-t}{\tau_{L d}}}
$$

And at the same time, the instantaneous voltage of reactor should be $u_{L}(t)$.

$$
u_{L}(t)=L_{d} \frac{d i_{L d}(t)}{d t}
$$

The limit of formula (39) is expressed as formula (40).

$$
\lim _{x \rightarrow 0^{+}} u_{L}(t)=\sqrt{6} U_{2} \sin \left(\frac{\pi}{3}+\alpha\right)
$$

There must own the expression as formula (41) under the safety consideration. 


$$
u_{L}(t) \leq U_{s 0}
$$

The minimum value of initial rectifier angle can be expressed by formula (40) and (41).

$$
\alpha_{\min }= \begin{cases}-\frac{\pi}{3}+\arcsin \frac{U_{s 0}}{\sqrt{6} U_{2}}, & 0 \leq \alpha \leq \frac{\pi}{6} \\ \frac{2 \pi}{3}-\arcsin \frac{U_{s 0}}{\sqrt{6} U_{2}}, & \frac{\pi}{6}<\alpha \leq \frac{7 \pi}{6}\end{cases}
$$

Formula (36) and (42) have given the limit conditions of rectifier angle when starting. If $\alpha_{\max }<\alpha_{\min }$, it would be failure to start. Others, to enhance the starting performance, i.e. to shorten the setting time, it requires the current $i_{L d}(t)$ to response at the most variation speed all the time, in which, it gives out the maximum variation of $\alpha$, namely $\Delta \alpha_{\max }$ expressed as formula (43).

$$
\Delta \alpha_{\max }= \begin{cases}-\frac{\pi}{3}-\alpha_{0}+\arcsin \frac{U_{s 0}+\sqrt{6} U_{2} \sin \left(\frac{\pi}{3}+\alpha_{0}\right)}{\sqrt{6} U_{2}}, & 0 \leq \alpha_{0}+\Delta \alpha_{\max } \leq \frac{\pi}{6} \\ \frac{2 \pi}{3}-\alpha_{0}-\arcsin \frac{U_{s 0}+\sqrt{6} U_{2} \sin \left(\frac{\pi}{3}+\alpha_{0}\right)}{\sqrt{6} U_{2}}, & \frac{\pi}{6}<\alpha_{0}+\Delta \alpha_{\text {max }} \leq \frac{7 \pi}{6}\end{cases}
$$

Where, $\alpha_{0}$ is the former trigger angle of rectifier, and $\Delta \alpha_{\max }$ is the maximum variation of $\alpha$ in the next rectifier period.

\subsubsection{Selection of Inverter Trigger Frequency}

The inverter trigger frequency $\omega_{1}$ is also an important control variable in the TPSPRIH system, the state of the equivalent load circuit is different under different $\omega_{1}$. When $\omega_{1}>$ $\omega$, the load circuit is in capacitive state, when $\omega_{1}<\omega$, it's in inductive state, and when $\omega_{1}$ $=\omega$, it's in resistive state that is called the resonant state.

From the formula (6), when the heated iron bar is in no difference in material and the size, the load circuit power consumption is only determined by load oscillation frequency because of $P_{\text {out }}$ being positively correlated with $\omega$. Therefore, if the frequency is selected to be much smaller, the decay speed of energy will be largely lowered, and it owns more advantageous to start. But concerned with the turn-off condition of thyristor, the equivalent load circuit must be in capacitive state, thus $\omega_{1}>\omega$ is a necessary condition during the starting process. Under the comprehensive consideration, the reference condition of inverter trigger frequency can be expressed as formula (44).

$$
\omega_{1} \geq 1.3 \omega
$$

\section{Simulation}

\subsection{The Range of Rectifier Angle}

The selected region of rectifier angle is shown as in Figure 6. It is respectively selected to the simulation value of equivalent resistance $R$ and equivalent inductance $L$ of load inductor in terms of formula (8) and formula (1), the simulation value of $I_{d o m i n}$ and $\omega$ refers to formula (33) and (44), the critical values $\alpha_{\max }$ and $\alpha_{\min }$ can be figured out in terms of formula (37) and formula (42), and then the curves of $\alpha_{\max }-\delta$ and $\alpha_{\max }-\delta$ can be plotted as in Figure 6. 


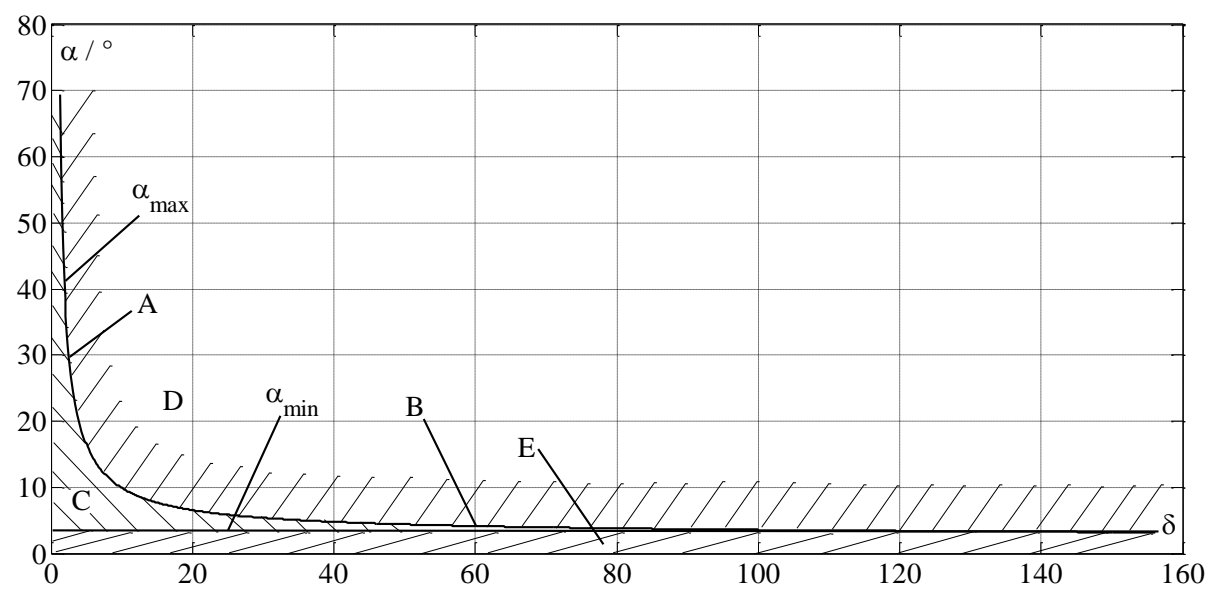

Figure 6. Variation of Curve $A-\Delta$

From Figure 6 , it can be seen that the curves $\alpha_{\max }-\delta$ and $\alpha_{\max }-\delta$ divide the plane into three parts. In which, the part $C$ is safe starting region of rectifier angle. When the rectifier angle is in the part $\mathrm{D}$, it can not be started any longer. The part $\mathrm{E}$ is non-safe starting region. A, B is exactly on the critical curve of $\alpha_{\max }$. When the load decay factor $\delta$ is smaller, the range of $\alpha$ is wide. With the change of the load parameters, $\delta$ increases much faster, and therefore, the range of $\alpha$ becomes an extremely narrow region.

\subsection{Simulation Experiment of Starting Process}

In the simulation experiment, the rated parameter of medium frequency power supply is set as $1000 \mathrm{KW} / 1000 \mathrm{~Hz}$, and it adapts MATLAB/Simulink as the simulation tool. The simulation parameters are set as $U_{2}=254 \mathrm{~V}, L_{d}=5 \mathrm{mH}, C=300 \mu \mathrm{F}, U_{0}=20 \mathrm{~V}, U_{H 0}=$ $500 \mathrm{~V}$. The response curve of varying process of load voltage is shown as in Figure 7.

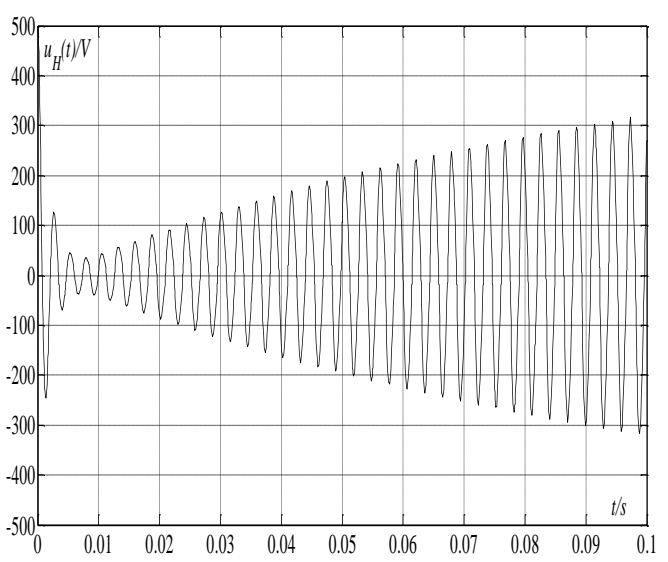

(a) Starting process of point $\mathrm{A}$

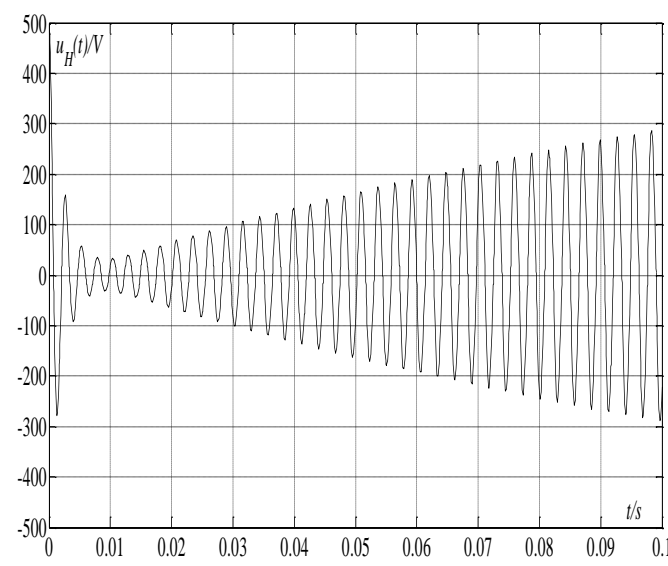

(b) Starting process of point B 


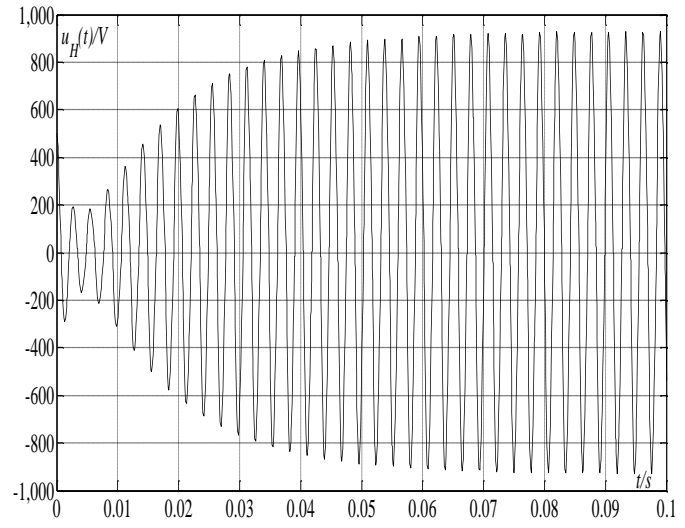

(c) Starting process in region $\mathrm{C}$

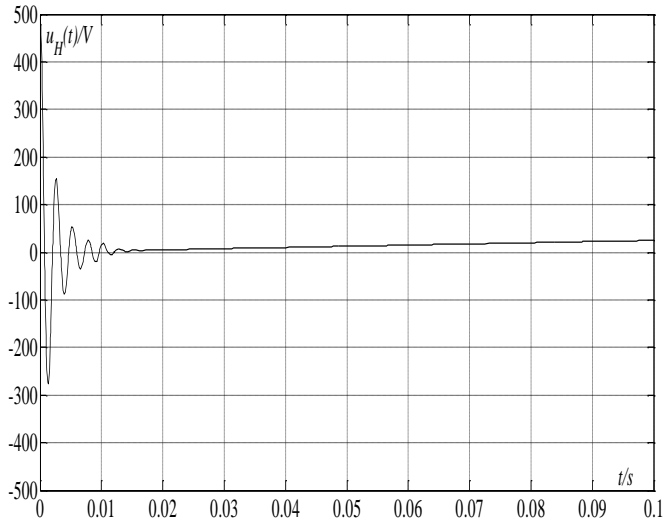

(d) Starting process in region $D$

Figure 7. Load Voltage Response in Starting Process

\subsection{Analysis of Simulation Result}

Figure 7 (a), (b) is respectively the load voltage response curve at point A and B in critical rectifier angle of different maximum in Figure 6. Obviously, both situations could start successfully with different $\alpha$, but in the starting process, the instantaneous value of load voltage is very close to the critical detecting value $U_{0}$ which is set in simulation. At the same time, the response speed gets slow, the regulation time of steady state gets longer in time. In addition, the decay speed of $\mathrm{A}$ is smaller than $\mathrm{B}$, but the starting process is similar because both of them are under critical states.

Figure 7 (c) is the response of state for $\mathrm{C}$ in Figure 6. It's in the starting region, also the rectifier angle could not reach the critical value, and therefore, the starting performance is superior to critical state.

Figure 7 (d) is the response of state for D in Figure 6. It's in the cannot-start region. Because the rectifier angle is lager than the critical value, the energy supply speed cannot reach the requirement, and at last, it gets the failure to start.

\section{Conclusions}

In the pre-charge starting of TPSPRIH, the starting characteristic on heavy load is determined by (1) initial current of DC side, (2) rectifier angle, and (3) initial trigger frequency of inverter under other conditions being same. All of them would influence the energy supplement, but the effect and start time are different. The upper limit of the initial energy in the system depends on the condition (1), and it mainly determines the circumstances before triggering. If the control of condition (2) cannot be regulated in time, it will affect the energy supply directly after the triggering, but a timely adjustment of rectifier angle can improve the response speed according to the current state and the next state of load. At the same time, the condition (3) will influence the commutation time of inverter directly, which means a matchless trigger frequency leads to a failed commutation of thyristor because of the complexity of starting process and the rapid change of parameters, and thus, it is necessary to own a sufficient margin. The three conditions mentioned above are expressed in explicit form, and from these conditions, it can obtain unique solution at different state time.

At present, the most of TPSPRIH adopt the digital control type, and it will be more convenient to express these constraints conditions with accurate value in engineering. Therefore, it can obtain a better control performance, which makes a contribution to the starting control about relevant AC-DC-AC power supply. 


\section{References}

[1] I. Yilmaz, M. Ermiş and I. Çadirci, "Medium-Frequency Induction Melting Furnace as a Load on the Power System", IEEE Transactions on Industry Applications, vol.48, no.4, (2012), pp.1203-1214.

[2] A. Namadmalan, S. Abedi, S. H. Hosseinian and J. S. Moghani, "Power quality improvement for single phase and three phase current source induction heating systems" Power Qual. Conf., PQC. 20101 st Power Quality Conferance Tehran, Iran, (2010).

[3] C. Saichol, K. Choyant and M. Veerapol, "Full-bridge current-fed inverter with automatic frequency control for forging application", IEEE TENCON 2004-2004 IEEE Region 10 Conference: Analog and Digital Techniques in Electrical Engineering, Chiang Mai; Thailand, (2004), pp.D128-131.

[4] J. Jordán, J.M Magraner, C. Cases, V. Esteve, E. Dede, E. Sanchis, E. Maset, A. Ferreres, J.B. Ejea and E. Gumbau, "Short-Circuit Critical Frequency for Induction Heating Parallel Resonant Inverters", 13th European Conference on Power Electronics and Applications, Barcelona, Spain, (2009).

[5] R. Fuentes, J. Juliet, F. Campaña, F. Ahumada, C. Silva and J. Estrada, "Comparative Analysis of Three Starting Methods for Parallel Resonant Current Source Inverter", 5th IET International Conference on Power Electronics, Machines and Drives, Brighton, United Kingdom, (2010).

[6] I. Wallace, A. Bendre, J.P. Nord and G. Venkataramanan, "A unity-power-factor three-phase PWM SCR rectifier for high-power applications in the metal industry", IEEE Transactions on Industry Applications, vol.38, no.4, (2002), pp.898-908.

[7] Q. Fu and M. Luo, "Technique of Starting Parallel Inverter Intermediate-Frequency Power Supply on Heavy Load", Transaction of Beijing Institute of Technology, vol.28, no.3, (2008), pp. 241-244.

[8] Z. Q. Meng, Y. Z. He and Y. Y. Peng, "A Study of the Starting Method of Magnetizing to the Load of SCR Medium-frequency Power Supply”, Transactions of China Electrotechnical Society, vol.18, no.2, (2003), pp. 57-61.

[9] M. G. Kim, G. Y. Park, D. H. Yoo and G. Y. Jeong, "High Power Full-Bridge DC-DC Converter using a Center-Tapped Transformer and a Full-Wave Type Rectifier", International Journal of Control and Automation, vol.7, no.4, (2014), pp.267-278.

[10] Y. Sun, Y. H. Wang, X. G. Yang and L. L. Pang, “A Novel Coil Shape for Transverse Flux Induction Heating", Transactions of China Electrotechnical Society, vol.29, no.4, (2014), pp.85-90.

[11] J. S. Gao, F. X. Liu and Z.H. Sun, "Research on a Network Model of Induction Heating Loads", Power Electronics, vol.43, no. , (2009)1, pp. 39-41.

[12] J. G. Li, Y. R. Zhong and Z. F. Ming, "A New Analysis and Design method of Load-Matching Static Characteristics for Voltage-Source Induction Heating", Transactions of China Electrotechnical Society, vol.21, no.11, (2006), pp.101-105.

[13] M. Xue, Q. X. Yang, Y. Li, X. Zhang and W. N. Liu, "The Study of Load Characteristic in Wireless Energy Transfer System Based on EM Coupling Resonance", Transactions of China Electrotechnical Society, vol.28, no.2, (2013), pp.28-34.

[14] Z. J. Zhang, X. F. Jin, H. M. Li and Y. L. Peng, "Research on Induction Heating Voltage Source Inverter Load Matching", Journal of North China Electric Power University, vol.37, no.5, (2010), pp. 52-56.

[15] J. W. Yue, H. M. Chen and Z. S. Wang, "Research on Ultra High Frequency Induction Heating Power Supply Inverter Load Topology”, Journal of Mechanical \& Electrical Engineering, vol.27, no. 6, (2010), pp. 73-77.

[16] G. Q. Fu, Y. H. Geng and E. H. Liu, "Two Kind of Startup Mode Design and Analysis of the Intermediate Frequency Power Supply”, Journal of Hebei University of Technology, vol.36, no.4, (2007), pp.48-51.

[17] Y. B. Han, "The Design of the Inductor Used in High-Frequency Induction Heating Equipment", M.S. of Zhengzhou University, (2013). 


\section{Authors}
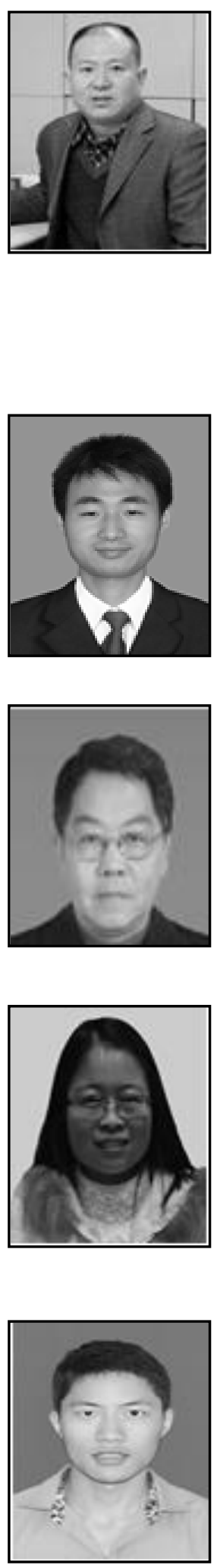

Sen-Lin Cheng, he received his B.S., M.S. and Ph.D. degrees in Detection Technology and Instrument, Automatic Control Theory and Application and Mechanical Manufacture and Automation from Chongqing University, China, in 1990, 1993 and 1999, respectively. He has been an Associate Professor and supervisor for master student in School of Automation, Chongqing University. He has also been a member of fuzzy information and engineering branch of ORSC and NDT Society. His research interests include power electronics, pattern recognition and image technology, detection technology and automation systems, remote sensing and control technology, and embedded systems as well as network control.

Hu Xu, he received his B.S. degree in Automation from Dalian Maritime University, China, in 2013. He is currently working toward the M.S. degree in Control Science and Engineering at Chongqing University. His research interests include power electronics, circuits and systems and control systems.

Bin Li, he received the B.S., M.S. and Ph.D. degrees in Industrial Automation, Automatic Control Theory and Application and Mechanical Manufacture and Automation from Chongqing University, China, in 1985, 1992 and 1999, respectively. He has been an Associate Professor and supervisor for master student in School of Automation, Chongqing University. His research interests include control theory, navigation, guidance and control.

Bu-Qing Liu, she received her B.S. degree in Detection and Control Technology from Nanchang Hangkong University, China, in 2013. She is currently working toward the M.S. degree in Control Science and Engineering at Chongqing University. Her research interests include tracking telemetering and command, wireless sensor networks and cognitive radio.

Chuan Wang, he received his B.S. degree in Automation from Chongqing University, China, in 2014. He is currently working toward the M.S. degree in control engineering at Chongqing University. His research interests include power electronics, circuits and systems. 